\title{
Assessment of Metabolites Content of Native Alcoholic Beverages Obtained from Jos, Nigeria
}

\section{Samuel Yusufu Gazuwa*, Jonathan Dingkwoet Dabak, Kiri Hashimu Jaryum, Segun Afolabi Olomu}

Department of Biochemistry, College of Health Sciences, University of Jos, Jos, Nigeria

Email address:

sygazuwa@gmail.com (S. Y. Gazuwa), gazuwas@unijos.edu.ng (S. Y. Gazuwa)

${ }^{*}$ Correspondence author.

\section{To cite this article:}

Samuel Yusufu Gazuwa, Jonathan Dingkwoet Dabak, Kiri Hashimu Jaryum, Segun Afolabi Olomu. Assessment of Metabolites Content of Native Alcoholic Beverages Obtained from Jos, Nigeria. International Journal of Food Science and Biotechnology.

Vol. 5, No. 4, 2020, pp. 97-101. doi: 10.11648/j.ijfsb.20200504.19

Received: November 3, 2020; Accepted: November 20, 2020; Published: December 16, 2020

\begin{abstract}
Burukutu, (BKT) Pito (PT) and Goskolo (GSK) are drinks consumed routinely within the area of study. Although consumption of GSK is illegal owing to its life threatening toxicity, people still consume it secretly. Whereas BKT and PT are locally prepared using unscientific methods in preparing the drinks, GSK is a mixture containing different compounds and chemicals. BKT and PT are more commonly ingested than GSK. Because methods applied during 'brewing' BKT and PT are not scientific, toxic metabolites might be generated. So, the drinkers are prone to their toxic effects. This work ascertained the presence and levels of unwanted metabolites in these drinks using Gas chromatographic-Mass spectrometric (GC-MS) technique. Metabolites could aggravate the toxicity of alcohol and alcohol-related disease conditions. Results, (\% relative abundance), showed the significant presence in BKTof6-Octadecanoic acid (38.51), Hexadecanoic acid (20.92), Isopentyl alcohol (12.27), 1,11,13-Octadecatriene (11.60) among others. In PT,9-Octadecanoic acid (39.40), Hexadecanoic acid (15.98), Carbonic acid methylpentyl ester (14.22), 9,17-Octadecadienal (6.37) were the major components. In GSK, 6-Octadecenoic acid (42.43), n-Hexadecanoic (17.51), 1-Butanol (16.52) 9 and 9,12-Octadecadienoic acid (10.72) were the dominant components. BKT, PT, and GSK are composed of compounds/metabolites that are organic in nature, with about $83 \%$ of them being organic acids. This leads to a decrease in $\mathrm{pH}$ thereby making the drinks acidic. It is concluded that the alcoholic beverages contain metabolites that predispose drinkers to metabolic or respiratory acidosis, due to drop in $\mathrm{pH}$. Furthermore, these metabolites could aggravate the toxicity of alcohol. Other components of the samples include ketones, different types of alcohols, aldehydes, derivatives of benzene and esters.
\end{abstract}

Keywords: Organic, Metabolites, Acids, Burukutu, Pito, Goskolo, Toxic

\section{Introduction}

Native beers (BKT and PT) are widely consumed as food (because it is thick, heavy, and nutritious) in the rural areas of northern Nigeria and in poor urban neighborhoods because it is more affordable than commercially brewed beer [1]. Also, native beers find relevance during cultural activities such as payment of dowry during marriage ceremonies, naming of children and even for religious purposes $[2,3]$ Solange et al., 2014). But these drinks are prepared under unscientific conditions and techniques. This could result in metabolic shift from a precursor to undesirable and harmful metabolites [4]. This work was designed to qualitatively and quantitatively assess the metabolites generated during preparation of native beers.

\section{Materials and Methods}

\subsection{Materials}

(GCMS-QP2010) SHIMADZU, Equipment. Stationary phase used was polyethylene glycol (PEG 400). Mobile phase used was helium gas.

Operating conditions $(G C)$ : Column oven temperature: $60^{\circ} \mathrm{C}$; Injection Temperature: $250^{\circ} \mathrm{C}$; Injection Mode: split; 
Flow control Mode: Linear Velocity; Pressure: 100.20kPa; Total Flow: $6.2 \mathrm{ml} / \mathrm{min}$; Column Flow: $1.61 \mathrm{ml} / \mathrm{min}$; Linear Velocity: $46.3 \mathrm{~cm} / \mathrm{sec}$; Purge Flow: $3.0 \mathrm{ml} / \mathrm{min}$; Split ratio: 1.0; High Pressure Injection: off, Carrier Gas Saver: off; Splinter Hold: off. Oven Temperature: At zero rate: temperature was $60^{\circ} \mathrm{C}$, Hold Time (minute): 5.0. At 5.0 rate: Temperature was $140^{\circ} \mathrm{C}$, Hold Time was 0.00 . At 15 rate: Temp was $280^{\circ} \mathrm{C}$ whereas Hold Time was 10.00 . Ion source Temperature: $200.00^{\circ} \mathrm{C}$, Interface Temperature: $250^{\circ} \mathrm{C}$, Solvent cut Time: $2.5 / \mathrm{min}$; Detector Gain Mode: Relative; Detector Gain: $0.00 \mathrm{Kv}$; Threshold: 4000 .

Operating conditions (MS): Start Time: $3.0 \mathrm{~min}$; End Time: $38.0 \mathrm{~min}$; ACQ Mode: Scan; Event Time: 0.50 seconds; Scan Speed: 769; Start m/z: 30.00; End m/z: 400.00.

\subsection{Methods}

Preparation of samples

Native beer samples were purchased from local drinking joints scattered in area of study. They were kept in plastic containers and stored in a refrigerator. Prior to analysis, refrigerated samples were removed and kept on table in open laboratory to change to liquid form. They were filtered threefold using filter paper; spun at $3000 \mathrm{rpm}$ for 2 hours in a refrigerated ultracentrifuge machine. The supernatant was separated from the pellet by careful decantation. Each supernatant was re-centrifuged using the same procedure above until a clear and transparent sample was obtained. Samples were then analysed using Gas chromatographic-Mass spectrometer (GCMS-QP2010) SHIMADZU Equipment.

\section{Results and Discussion}

Table 1. Gas Chromatographic-Mass Spectrometric analysis results of Burukutu.

\begin{tabular}{llll}
\hline \multirow{2}{*}{ Peak number } & Retention time & $\begin{array}{l}\text { Chemical formula of } \\
\text { Compound }\end{array}$ & Name of \\
\cline { 2 - 3 } & (minutes) & $\mathrm{C}_{5} \mathrm{H}_{12} \mathrm{O}$ & Compound \\
\hline 1 & 3.34 & $\mathrm{C}_{2} \mathrm{H}_{4} \mathrm{O}_{2}$ & Isopentyl alcohol \\
2 & 3.67 & $\mathrm{C}_{2} \mathrm{H}_{8} \mathrm{O}_{2} \mathrm{Si}$ & Ethylic acid \\
3 & 4.27 & $\mathrm{C}_{4} \mathrm{H}_{10} \mathrm{O}_{2}$ & Dihydroxydimethylsilane \\
4 & 5.45 & $\mathrm{C}_{8} \mathrm{H}_{10} \mathrm{O}$ & 2.09 \\
5 & 14.52 & $\mathrm{C}_{16} \mathrm{H}_{32} \mathrm{O}_{2}$ & 2,3- Dihydroxybutane \\
6 & 28.33 & $\mathrm{C}_{18} \mathrm{H}_{34} \mathrm{O}_{2}$ & Benzeneethylalcohol \\
7 & 29.53 & $\mathrm{C}_{18} \mathrm{H}_{32}$ & Hexadecanoic acid \\
8 & 29.79 & $\mathrm{C}_{37} \mathrm{H}_{74} \mathrm{NO}_{8} \mathrm{P}$ & 6.48 \\
9 & 30.38 & $\mathrm{C}_{20} \mathrm{H}_{40} \mathrm{O}_{2}$ & 6-Octadecanoic acid \\
10 & 30.78 & $\mathrm{C}_{57} \mathrm{H}_{104} \mathrm{O}_{6}$ & 1,11,13-Octadecatriene \\
11 & 31.51 & & AH1,2AHPOEE \\
\hline
\end{tabular}

H12AHPOEE = Hexadecanoic acid, 1-[[[(2 aminoethoxy)hydroxyphosphinyl]oxy]methyl]-1,2-ethanediyl ester.

*2,3B9OP9O = 2,3-Bis[(9)-9-Octadecenoyloxy]propyl-9-octadeconate.

Table 2. Gas Chromatographic-Mass Spectrometric analysis results of Pito.

\begin{tabular}{lllll}
\hline \multirow{2}{*}{ Peak number } & Retention time & Chemical formula & Name of & Area (\%) \\
\cline { 2 - 4 } (minutes) & of Compound & Compound & Carbonic acid, methyl pentyl ester \\
\hline 1 & 3.317 & $\mathrm{C}_{7} \mathrm{H}_{14} \mathrm{O}_{3}$ & 2,3 -Butanediol & 5.46 \\
2 & 4.52 & $\mathrm{C}_{4} \mathrm{H}_{10} \mathrm{O}_{2}$ & O-Hydroxytoluene & 4.77 \\
3 & 13.676 & $\mathrm{C}_{7} \mathrm{H}_{8} \mathrm{O}$ & Benzene ethyl alcohol & 3.64 \\
5 & 14.513 & $\mathrm{C}_{8} \mathrm{H}_{10} \mathrm{O}$ & Hexadecanoic acid & 15.98 \\
6 & 28.283 & $\mathrm{C}_{16} \mathrm{H}_{32} \mathrm{O}_{2}$ & 9-Octadecanoic acid & 39.4 \\
7 & 29.48 & $\mathrm{C}_{18} \mathrm{H}_{34} \mathrm{O}_{2}$ & $9,17-$ Octadecadienal & 6.37 \\
9 & 29.968 & $\mathrm{C}_{18} \mathrm{H}_{32} \mathrm{O}$ & Arachidic acid & 2.05 \\
10 & 30.374 & $\mathrm{C}_{20} \mathrm{H}_{40} \mathrm{O} 2$ & $1,2-15,16$-Diepoxyhexadecane \\
\hline
\end{tabular}

Table 3. Gas Chromatographic-Mass Spectrometric analysis results of Goskolo.

\begin{tabular}{lllll}
\hline Peak & Retention time & Chemical formula of Compound & Name of Compound \\
\cline { 1 - 2 } number & (minutes) & & Area (\%) \\
\hline 1 & 3.42 & $\mathrm{C} 5 \mathrm{H} 12 \mathrm{O}$ & 1 -Butanol & 16.52 \\
3 & 3.75 & $\mathrm{C}_{2} \mathrm{H}_{8} \mathrm{O}_{2} \mathrm{Si}$ & Dihydroxydimethylsilane & 0.72 \\
4 & 5.04 & $\mathrm{C}_{5} \mathrm{H}_{10} \mathrm{O}_{3}$ & Propanoic acid, 2-hydroxy-ethyl ester & 0.05 \\
5 & 11.09 & $\mathrm{C}_{3} \mathrm{H}_{8} \mathrm{O}_{3}$ & $1,2,3$-propanetriol & 2.15 \\
6 & 14.51 & $\mathrm{C}_{9} \mathrm{H}_{12} \mathrm{O}$ & Benzene ethanol & 0.14 \\
\hline
\end{tabular}




\begin{tabular}{lllll}
\hline Peak & Retention time & \multirow{2}{*}{ Chemical formula of Compound } & Name of Compound & Area (\%) \\
\hline number & (minutes) & & 6-Octadecenoic acid & 42.43 \\
8 & 29.49 & $\mathrm{C}_{18} \mathrm{H}_{34} \mathrm{O}_{2}$ & 9,12 -Octadecadienoic acid & 10.72 \\
9 & 29.75 & $\mathrm{C}_{18} \mathrm{H}_{32} \mathrm{O}_{2}$ & *PDAHHMEE & 1.76 \\
10 & 30.38 & $\mathrm{C}_{18} \mathrm{H}_{36} \mathrm{O}_{4}$ & Eicosanoic acid & 3.56 \\
11 & 30.76 & $\mathrm{C}_{20} \mathrm{H}_{40} \mathrm{O}_{2}$ & cis-9-Hexadecenal & 4.45 \\
\hline
\end{tabular}

Pentadecanoic acid-2-hydroxy-1-(hydroxymethyl) ethyl ester.

\section{Discussion}

This work sought to evaluate the presence and levels of volatile organic metabolites and compounds in Burukutu (BKT) and Pito (PT) and also in Goskolo (GSK). GSK isa banned drink owing to its lethal attributes. Ethyl alcohol is the physiologically active component of alcoholic beverages. However, congeners do remain as fractions of brewing. Even if quantitatively small, congeners play crucial role in the social use and of the alcohol abuse. They may be volatile compounds such as alcohols, acids, aldehydes, ketones and esters. Carbohydrates, tannins, phenols, metals, colouring agents, minerals, histamines and pharmacologically active components constitute other components. [5]. Ethyl acetate, ethyl formate methyl alcohol, acetaldehyde, and the small aliphatic alcohols (n-propyl alcohol, isobutyl alcohol, nbutanol constitute volatile congeners make up the major volatile congener content of beers, wines and distilled spirits.

Consumption of native beers is common among residents of the area of study because they are relatively cheaper, culture friendly and readily available compared to branded factory-based lager beers. [4] reported that BKT samples obtained from B/Ladi contained trifluoroacetic acid, 2methyl nonadecane and 2-tetradecen-1-ol. PT contained dichloroacetic, pentadecanoic acid,1-hexadecanol,1tetracosanol, methyl tetradecanoate.

Results (\% relative abundance), from this study showed the significant presence in BKTof6-Octadecanoic acid (38.51), Hexadecanoic acid (20.92), Isopentyl alcohol (12.27), 1,11,13-Octadecatriene (11.60) among others. Isopentyl alcohol is a toxic compound. It targets the central nervous system (CNS), respiratory system, eye and skin. It is a product of gut microbial fermentation. It is the major higher chain alcohol in alcoholic beverages. It induces expression of CYP3A and CYT2E1 in human liver [6]. Another metabolite in BKT is Linolenic acid (Octadecatriene). It is particularly implicated in causing cardiovascular disease [7]. Although retention times of compounds may be similar or the same, it is not a fixed property of a component but result of a system which contributes to the Retention Time (RT). RT does not guarantee a conclusive identification of a compound present in a sample. However, it gives a more reliable insight into the identity of metabolites when coupled with (GC-MS).

There were no metabolites in BKT with identical retention times albeit some were very similar. For example, 6Octadecanoic acid with retention time (RT) of 29.53and 1,11,13-Octadecatriene with RT of 29.79. So also Arachidic acid with RT of 30.78 and Hexadecanoic acid, 1-[[[(2 aminoethoxy) hydroxyphosphinyl]oxy]methyl]-1,2ethanediyl ester with 30.38 .

In PT, retention times of most of the metabolites were generally similar ranging between 29.48 to 30.76 . Metabolites present in it included 9-Octadecanoic acid, 9,17Octadecadienal, Arachidic acid, Carbonic acid methylpentyl ester, Benzene ethyl alcohol, 2,3-butanediol, OHydroxytoluene and 1,2-15,16-diepoxy hexadecane. Carbonic acid methylpentyl ester causes behavioral analgesia; attacks a segment of the central nervous system (CNS) including aquatic life with long side effect. PT is the major cause of illness among drinkers. It darkens the skin, and causes oedema and weakness. Its ethyl alcohol content is less than that in BKT [8].

As for GSK, the range of RT was between 29.29 and 31.38 (2.09). Some compounds present include 6Octadecanoioic acid, 9,12-Octadecanoic acid, Benzene alcohol, Pentadecanoic acid-2-hydroxy-1-(hydroxymethyl) ethyl ester, 1,2,3-propanetriol and Hexadecanoic acid. Hexadecanoic acid causes the generation of ketone bodies in excess of acetyl-coA, inhibits mouse pronuclear and 2 stage embryo development [9]. It raises the level of low density lipoprotein more than other saturated fats. High consumption of palmitate predisposes to cardiovascular disease. One unique metabolite in GSK is 1-Butanol. It is implicated in eye skin, central nervous system ailments [10]. Benzene ethyl alcohol is toxic and lethal to hepatocytes whose toxicity is not mitigated by ethyl alcohol [11]. Chronic ethanol ingestion exacerbates benzene myelotoxicity and reduces the urinary excretion of benzene metabolites in CD-1 mice [12].

BKT, PT and GSK are composed of diverse metabolites/compounds most of which are organic acids. Among the major metabolites in all the drinks, some had identical RTs. In BKT, Hexadecanoic acid, 1-[[[(2 aminoethoxy) hydroxyphosphinyl] oxy] methyl]-1,2ethanediyl ester had RT of 30.38 which is the same with Pentadecanoic acid-2-hydroxy-1-(hydroxymethyl) ethyl ester in GSK. Further, Eicosanoic acid in GSK had identical RT of 30.76 with 1,2-15,16-diepoxyhexadecane in PT. Arachidic acid in BKT had same RT with cis-9-hexadecenal in GSK with RT value of 31.51 .

In normal homeostasis, there is a need for a balanced state of equilibrium. The physiological $\mathrm{pH}$ for humans is 7.4. Since the living system is dynamic, there is the requirement to control the redox status (acidity and alkalinity) of cells. This is achieved by using appropriate buffers which help to 
reasonably control the $\mathrm{pH}$ over a narrow range of changes compatible with life. Metabolic acidosis is a disturbance in the homeostasis of acidity of the plasma. If serum hydrogen ion concentration increases owing to processes, then it is a distinct acidosis. Acidosis is either respiratory which involves some changes in carbon iv oxide or metabolic which is influenced by bicarbonate. Metabolic acidosis increases the hydrogen ion concentration in the body particularly systemic circulation. Increased production of acid, acid ingestion, renal or gastrointestinal bicarbonate losses and decreased excretion of acid constitute etiologies of metabolic acidosis [13].

To be neutral, number of cations must balance anions. Sodium is the primary cation in the plasma. So, it is balanced by the sum of the bicarbonate and chloride ions which represents the anion gap. However, lactate and acetoacetate are unmeasured anions that are the main contributors to metabolic acidosis. Anion gap metabolic acidosis is frequently due to anaerobic metabolism and lactic acid accumulation. If the lungs are not able to remove carbon iv oxide produced, respiratory acidosis ensues. As a consequence, the $\mathrm{pH}$ of the blood and other fluids decrease making them too acidic and incompatible to homeostasis of the cell. In this work, at least $75 \%$ of compounds and metabolites present in samples of BKT, PT and GSK are organic acids. Hence, the $\mathrm{pH}$ of the blood, plasma, serum and other body fluids of drinkers reduce. As a result, drinkers are at risk of metabolic acidosis and its consequences. Mean amount of native beers consumed in a day by males and females drinkers in the area of study stands at $3,745 \mathrm{dm}^{-3}$ and $2,946 \mathrm{dm}^{-3}$ respectively [8].

Some compounds and metabolites in BKT and PT could be of benefit to the body. BKT contains Octadecanoic acid (Stearic acid) lowers the concentration of low density lipoprotein (LDL) cholesterol relative lauric and palmitic acids. LDL is harmful to the body because of its low density, which makes it to easily deposit on the inner surface of the blood vessels thereby reducing their diameter. This leads to arthrosclerosis; a lethal disease condition that overworks the heart. BKT contains vitamins, iron, magnesium, manganese, phosphorus, calcium, $26.7 \mathrm{~g}$ starch, and $5.9 \mathrm{~g}$ of protein per liter [14]. Eicosanoic acid is a component of oily fruits such as coconut but in small quantity [15]. In addition, cis-9-hexadecanal has potential antimelanogenic antifungal attributes. It targets cell wall organisation, critical Growth Factor \& virulence in Aspergillus fumugatus [16]. It contains almost all essential amino acids in required proportions except cystine and tryptophan which have been completely destroyed by heat during boiling [17].

PT is particularly enriched with nutrient elements such as $\mathrm{Cl}, \mathrm{Mg}$, and $\mathrm{K}$. Apart from serving as an inebriating drink, it is important in fulfilling cultural norms and values including such as marriages, naming and burial ceremonies, parties, and other social gatherings [18]. The elemental composition in this native beverage is crucial due to its nutritional value especially mineral elements as well as estimation of toxic levels [18]. PT can serve as functional food, owing to its antioxidant capabilities in addition to its gross energy content [19].

As for GSK, there has never been documented evidence about its beneficial attributes to the body. Consequently, its preparation and consumption has been banned by Plateau State Government, Nigeria.

\section{Conclusion}

It is concluded that BKT, PT, and GSK are composed of compounds and metabolites that are organic in nature. About $83 \%$ of them are organic acids, making the drinks acidic. This predisposes drinkers to metabolic or respiratory acidosis, due to drop in $\mathrm{pH}$. Drinkers are exposed to the toxicities of metabolites albeit BKT and PT contain beneficial mineral elements and other nutrients. Other components of the samples include ketones, different types of alcohols, aldehydes, derivatives of benzene and esters.

\section{References}

[1] Gazuwa, S. Y., Dabak, J. D., and Ubom G. A. (2006). Iron Contamination of Two Local Alcoholic Drinks. Journal of Medicinein the Tropics, 8 (1): 33-38.

[2] Gazuwa, S. Y., Dabak, J. D., and Ubom, G. A. (2008). Contaminants in local alcoholic beverages: zinc and manganesecontamination. International Journal of Biological and Chemical Sciences2 (4): 411-416.

[3] Solange, K. G, Fokou G, Dje, K. M., and Bonfoh B. (2014). Review on African traditional cereal beverages. American Journalof Research Communication; Vol 2 (5): 1-51.

[4] Gazuwa, S. Y., and Denkok, Y. (2017). Organic Metabolites, alcohol content and Microbial Contaminants in SorghumbasedNative Beers Consumed in Barkin Ladi Local Government Area, Nigeria. Journal of Scientific Research and Reports; 17 (4): 1-8. Article no, JSRR. 38124. ISSN: 2320-0227.

[5] Adriana, J, Harald, J, Volker A., Stefan P. A. M. Fárr, L. H, and Schiopu, A. (2010). Volatile congeners in alcoholic beverages: analysis and forensic significance. Romanian Journal of Legal Medicine; (18): 26-270. DOI: 10.4323/rjlm.2010.265.

[6] Kostrubsky V. E., Strom S. C. W, Wood, S. G, Wrighton, S. A, and Sinclair, J. F. (1995). Ethanol and isopentanol increase CYP3A and CYP2E in cultures of human hepatocytes. Arch Biochem Biophys; 2 (2): 516-520.

[7] An, P., Mu, C., Rajiv, C., Jason H., Wu, Y., Qi, S, Hanna. C., Darush, M. and Frank B. H. (2012). $\alpha$-Linolenic acid of CVDs: a systemic review and meta-analysis. The American Journal of Clinical Nutrition; 96 (6): 1262-1273.

[8] Gazuwa, S. Y. (2014). Toxicants in native beers locally prepared within Jos metropolis. Doctor of Philosophy (Ph. D) thesis in Biochemistry, University of Jos, Nigeria.

[9] United State Environmental Protection Agency (2005). Provisional peer reviewed toxicity values for Hexadecanoic acid. CASRN; 57: 10-13. 
[10] Segal D., Bale A. S., Philips L. J., Sasso A., Scholosser, P. M., Starkey, C. and Makris, S. L. (2020). Issues in assessing thehealth risks of n-Butanol. Journal of Applied Toxicology; 40 (1): $72-86$

[11] Bloch, P., Kulig, A.; Paradowski M. and Wybrzak-Wrobel T. (1990). Toxicodynamics of benzene, ethyl alcohol and benzeneplus ethyl alcohol based on the histopathological examination of selected organs in rats. Pol J Occup Med; 3 (1): 69-82.

[12] Giorgio M, Anna, F. C.; Teresa, C. and Lungi (2003). Prolonged ethanol ingestion enhances benzene myelotoxicity and lowersurinary concentration of benzene metabolites level in CD-1 male mice. Toxicological Sciences; 75 (1): 16-24.

[13] MacKenzie, B. and Derek, J. S. (2020). Metabolic Acidosis. In: StatPearls [Internet]. Treasure Island (FL): StatPearlsPublishing; 2020 Jan. 2020 Jul 25.

[14] Taofeek, O. A., Ganiyat, A. I., Oluwayemisi, B. O., Azeemat, T. A., Aisha, O. M. and Fausat L. K. (2014). Sorghumbasedalcoholic beverage, Burukutu perturbs the redox status of the liver of male rats. Food Sci Nutr; 2 (5): 591-596.
[15] Akoh, C. C., and Min, D. B. "Food lipids: chemistry, nutrition, and biotechnology" 3rd ed. 2008, pp. 1-18.

[16] Shanu, H., Lovely, G., Jata, S., Alok, K. G, and Pooja, V. (2020). c-9-hexadecenal, a natural compound targeting cell wallorganisation, critical growth factor \& virulence of Aspergillus fumugatus. ACS Omega; 5 (17): 1007-10088.

[17] Odetokun, S. M. (2006). Chemical and nutritive values of burukutu (a Nigerian beverage). Food and Nahrung; 41, (6): 13.

[18] Aaron, N. A., Apori, N., and Samuel, A. B. (2014). Analysis of essential elements in pito-a cereal food drink and its brandsby the single-comparator method of neutron activation analysis. Food Sci Nutr; 2 (3): 230-235.

[19] Taofeek, O. A., Ganiyat, A. I., Abdulwasiu, O. A., Folakemi, A. A., Shakirat, A. A., Simiat, M. O., Simiat, O. J, and Oyelolu (2014). Nutritional andantioxidantdispositions of sorghum/millet-based beverages indigenous to Nigeria. Food Sci Nutr; 2 (5): 597-604. 